\title{
Clima organizacional en funcionarios públicos de la Secretaría de Gobierno de Pasto $^{14}$
}

\author{
David Román Bárcenas Enríquez \\ Psicólogo \\ Universidad de Nariño, Colombia \\ Correo electrónico: davidromanbarcenas@gmail.com
}

\section{Edwin Andrés Pastás Cuayal}

Psicólogo

Universidad de Nariño, Colombia

Correo electrónico: andresck6@gmail.com

\section{María Alejandra Pantoja Montero \\ Eespecialista en Psicología de las Organizaciones Universidad Católica de Colombia Correo electrónico: alepantoja96@hotmail.com}

Recibido: $20 / 01 / 2020$

Evaluado: $13 / 03 / 2020$

Aceptado: 20/03/2020

\section{Resumen}

La investigación tuvo como objetivo evaluar el Clima Organizacional (CO) en 85 funcionarios públicos de la Secretaría de Gobierno de la ciudad de Pasto (Colombia). El estudio fue de carácter cuantitativo de tipo descriptivo transversal y la información se recolectó a través de la aplicación de la escala tipo Likert denominada Escala de Clima Organizacional (EDCO; Quevedo, Echeverri \& Sanabria, 1999), con una confiabilidad $=.853$, determinada por el coeficiente de Alpha de Cronbach. El nivel del C0 tuvo un valor promedio de 143. Las dimensiones con puntuaciones más altas fueron: estilo de dirección 22.1 y relaciones personales 20.1; con puntuación más bajas: sentido de pertenencia 14.3, valores colectivos 14.4 y estabilidad 14.6. Se concluye que los funcionarios se sienten medianamente satisfechos en la Secretaría de Gobierno; sin embargo, se encontró una baja satisfacción en la percepción de estabilidad laboral, los beneficios de salud recibidos y la asignación salarial.

Actitudes, clima organizacional, entidad pública, funcionario público.

14 Para citar este artículo: Bárcenas-Enríquez D.R, Pastás-Cuayal, E.A., y Pantoja-Montero, M.A. (2021). Clima organizacional en funcionarios públicos de la Secretaría de Gobierno de Pasto. Informes Psicológicos, 21(1), $217-229$ http://dx.doi.org/10.18566/infpsic.v21n1a14 


\title{
Organizational climate in public officials of the Ministry of Government of Pasto
}

\begin{abstract}
The objective of the research was to evaluate the Organizational Climate $(\mathrm{OC})$ in 85 public officials of the Government Secretariat of the city of Pasto (Colombia). The study was of a quantitative, descriptive, cross-sectional nature and the information was collected through the application of the Likert-type scale called Organizational Climate Scale (EDCO; Quevedo, Echeverri \& Sanabria, 1999), with a reliability $=.853$, determined by Cronbach's Alpha coefficient. The OC level had an average value of 143 . The dimensions with the highest scores were management style 22.1 and personal relationships 20.1; those with the lowest score, sense of belonging 14.3, collective values 14.4 and stability 14.6. It is concluded that the civil servants feel moderately satisfied in the Secretary of Government although a low satisfaction was found in the perception of job stability, the health benefits received and the salary allocation.
\end{abstract}

Keywords Attitudes, organizational climate, public entity, public official.

\section{Clima organizacional em funcionários públicos da secretaria do Governo de Pasto}

Resumo

0 objetivo da pesquisa foi avaliar o Clima Organizacional (CO) em 85 funcionários públicos da Secretaria de Governo da cidade de Pasto (Colômbia). 0 estudo foi de natureza quantitativa descritiva transversal e as informações foram coletadas por meio da aplicação da escala do tipo Likert denominada Escala de Clima Organizacional (EDC0; Quevedo, Echeverri \& Sanabria, 1999), com confiabilidade = 0.853 determinada pelo coeficiente Alpha de Cronbach. 0 nível de $\mathrm{CO}$ teve valor médio de 143. As dimensões com maiores pontuações foram: estilo de direção 22.1 e relações pessoais 20.1; com notas mais baixas: sentimento de pertença 14.3 valores coletivos 14.4 e estabilidade 14.6. Concluise que os funcionários se sentem moderadamente satisfeitos na Secretaria de Governo; no entanto, foi encontrada baixa satisfação na percepção da estabilidade no emprego, nos benefícios de saúde recebidos e na atribuição salarial.

\section{Palavras chave}

Atitudes, clima organizacional, entidade pública, funcionário público. 


\section{ntroducción}

Tanto las entidades de orden privado como público, están constituidas por factores como: la estructura física y organizacional, las políticas, los recursos, los colaboradores, entre otros (García, 2009), reconociendo principalmente al capital humano como un activo fundamental para la organización (Garzón, 2005; Pérez \& Castillo, 2016) y como un elemento clave que incide en la productividad, la economía, el alcance de las metas y el desarrollo de la organización en general (Goncalves, 2000; Montoya \& Boyero, 2016).

Por lo anterior, teniendo en cuenta los cambios y desafíos en el contexto laboral (Bravo, 2009), la mayoría de las empresas invierten sus recursos en el área de la gestión humana (Torres, 2015), buscando fortalecer, mantener y desarrollar al capital humano mediante estudios y programas relacionados con su comportamiento en las organizaciones, en pro de la competitividad, la productividad de las empresas y el bienestar de los colaboradores (Betancourt \& Caballero, 2010).

Respecto a la gestión humana, se ha atribuido al Clima Organizacional (CO) como un elemento importante en el contexto laboral, permitiendo comprender que las percepciones de los colaboradores respecto a su entorno laboral, determinan el comportamiento hacia su trabajo (Chiavenato, 2001), siendo así que las cogniciones, actitudes y conductas pueden afectar tanto positiva como negativamente en los objetivos, los logros, el desarrollo de las organizaciones y el desempeño de los mismos.

En referencia al $\mathrm{CO}$, se han identificado tres variables transversales que son comunes e interactúan entre sí: la estructura de la organización, los procesos organizacionales y los comportamientos, que, como resultado de su interrelación, pueden generar un clima tanto favorable como no favorable (Gómez, 2004). Un clima organizacional adecuado permite que la entidad sea permeable a los cambios que se presenten en todos sus procesos, lo que sugiere que el trabajar por un buen $\mathrm{CO}$ es beneficioso y de gran provecho para cualquier empresa (Chaves et al., 2017).

Para orientar la investigación, entre los estudios sobre el clima organizacional se encuentran: la investigación realizada en España a 303 trabajadores de organizaciones deportivas conformada por tres sectores: públicos, privados y asociados, encontrando que, en el sector público, existen puntuaciones más bajas respecto al clima en general comparándolo con los otros dos sectores, especialmente en las dimensiones de motivación, seguridad, formación, recursos y satisfacción laboral (Escamilla, Núñez \& Gómez 2016).

Otro estudio realizado a 561 trabajadores de hospitales de alta complejidad en Chile, encontró que las dimensiones con mayor puntuación favorable hacia el CO fueron: identidad, motivación y responsabilidad; por el contrario, la comunicación, la organización, los materiales y la administración del conflicto, obtuvieron menor puntuación (Bustamante, Gradón \& Lapo 2015). 
En el estudio realizado a 200 trabajadores de la Unidad de Gestión Educativa Local № 03 del sector público en Perú, se concluyó que las dimensiones bienestar, recompensa y retroalimentación se ubican por debajo del promedio de satisfacción del CO (Charry, 2018); asimismo, el estudio realizado con 30 profesionales de la salud un hospital público de Lima, concluyó que existe una correlación positiva entre el CO y Burnout, sugiriendo realizar evaluaciones periódicas, mínimo cada seis meses, con el fin de establecer programas preventivos de salud (Vizcarra, Llaja, Limo \& Talavera, 2015).

Según Sanabria (2016), en el territorio colombiano, a pesar de las investigaciones realizadas respecto al empleo público (citadas por Salas, Ortiz \& Márquez, 2016) y la normatividad establecida principalmente en la Ley 909 del 2004, se han evidenciado falencias respecto a la calidad de vida laboral de los funcionarios y los beneficios recibidos, siendo necesarios mecanismos de inspección y control por parte de las entidades encargadas de realizar la administración y vigilancia en este contexto, como la Comisión Nacional del Servicio Civil (CNSC) y el Estado. Todo esto debido a que el empleo público y la gestión del talento humano en el contexto público se considera como unas de las bases fundamentales para el Gobierno nacional, recomendando que se lleven a cabo acciones que promuevan ambientes de trabajo adecuados que resalten la labor de los servidores públicos, a través del diseño, evaluación e intervención al menos una vez por año, según sus necesidades.

En las investigaciones realizadas en Colombia se destaca la desarrollada con servidores públicos de la alcaldía de
Palestina, Pereira, la cual mostró que la Secretaría de Gobierno requiere mayor atención respecto a la percepción del ambiente laboral, en comparación con las otras secretarías, ya que se encontraron niveles bajos de satisfacción en las dimensiones: relaciones en el trabajo, salario y beneficios; lo que, a su vez, pudo incidir sobre la puntuación general obtenida del CO en la entidad (Cardona, 2016).

En Nariño (Colombia), el estudio realizado a colaboradores del sector público del Instituto Colombiano de Bienestar Familiar ICBF arrojó que, en las dimensiones del CO, el grado de satisfacción fue promedio a excepción de la retribución y los valores que puntuaron bajo; sin embargo, las condiciones del contexto pueden ser cambiantes, lo que requiere realizar acciones constantes de fortalecimiento para mantener un clima adecuado (Bacca \& Bravo, 2013). Así mismo, en el estudio aplicado en 144 funcionarios de la Secretaría de Educación y Cultura de Nariño, se concluyó que el CO incide sobre la motivación del personal y el desempeño laboral, por lo tanto, se debe articular la organización con el factor humano, siendo necesario que las entidades cuenten con mecanismos de evaluación periódica para el fortalecimiento continuo de la organización (Salas \& Ortiz, 2008).

Finalmente, debido a que actualmente no existe una evaluación o estudios sobre el clima organizacional en la Secretaría de Gobierno del municipio de Pasto, es importante la realización de esta investigación puesto que, al conocer las percepciones de los funcionarios hacia su entorno laboral, se pueden evidenciar las distintas dinámicas de la entidad en aspectos como liderazgo, trabajo en equipo, relaciones interpersonales y satisfacción, 
lo que, a su vez, son elementos que componen el $\mathrm{CO}$ e inciden sobre la gestión humana.

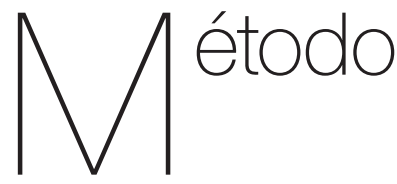

\section{Tipo de estudio}

Se realizó un estudio cuantitativo de carácter descriptivo transversal, el cual tuvo como objetivo identificar las actitudes de los funcionarios públicos de la Secretaría de Gobierno de Pasto hacia el clima organizacional; además, los datos fueron recolectados en el mes de diciembre del año 2019, en una sola ocasión.

\section{Participantes}

La población objeto de estudio estuvo constituida por funcionarios de la Secretaría de Gobierno de Pasto, ubicados en la oficina principal, la cual concentra al mayor número de funcionarios adscritos a la entidad, y distribuidos en cuatro dependencias: Subsecretaría de Justicia y Seguridad, Subsecretaría de Convivencia y Derechos Humanos, Oficina de Asesoría Jurídica y Subsecretaría de Control.

De los 109 funcionarios adscritos a la sede principal, se aplicó la fórmula para calcular la muestra en estudios descriptivos con poblaciones finitas (Aguilar, 2005), estableciendo finalmente un muestreo aleatorio simple, y se seleccionaron a 85 funcionarios de planta temporal y contratistas de la Secretaría de Gobierno de Pasto, descartando a las dependencias: Casas de Justicia, Comisarías de Familia, Corredurías, Inspecciones de Policía, de Control y de Urbanismo, por ser dependencias disgregadas y adscritas a la Secretaría de Gobierno que prestan sus servicios a la entidad aunque con una estructura administrativa propia.

Para el cálculo de la muestra, se tuvo en cuenta: el tamaño de la población ( $N$ = 190), el nivel de confianza $(Z=2)$, la variabilidad del parámetro $(p q=.25)$ y el error muestral máximo $(d=.05)$.

\section{Instrumento}

Para recolectar la información se utilizó un instrumento tipo Likert denominado Escala de Clima Organizacional (EDCO; Quevedo, Echeverri \& Sanabria, 1999). La escala EDCO cuenta con un proceso psicométrico de plan de prueba, formulación, creación y validación de ésta; teniendo en cuenta los criterios de confiabilidad y validez, tiene una confiabilidad alta (Alpha de Cronbach $=.853$ ) según los datos del estudio, lo que sugiere que la prueba es efectiva para medir el CO.

El cuestionario está conformado por 40 ítems, agrupados en 8 dimensiones: relaciones personales, estilo de dirección, sentido de pertenencia, retribución, disponibilidad de recursos, estabilidad, claridad y coherencia de dirección, y valores colectivos. Para la calificación del $\mathrm{CO}$, a cada respuesta de los reactivos se le asignó un valor numérico: las puntuaciones de 40 a 93 representaron un nivel de satisfacción bajo del CO; de 94 
a 147 un nivel de satisfacción promedio y de 148 a 200 un nivel de satisfacción alto (Quevedo et al., 1999).

\section{Procedimiento}

Los funcionarios seleccionados aleatoriamente fueron invitados a participar de manera voluntaria en el estudio, para contar con su consentimiento, acentuando sobre la normatividad de la Ley 1090 de 2006 en Colombia, referente a la confidencialidad en el tratamiento de los datos, la investigación científica, la ética y los beneficios sobre los colaboradores y la organización respecto hacia el $\mathrm{CO}$; diligenciando satisfactoriamente el documento mencionado.

Posteriormente, se realizó la aplicación de la escala a los 85 funcionarios seleccionados de la Secretaría de Gobierno de Pasto. Se llevó a cabo la tabulación de los datos y se hizo el análisis de la información obtenida mediante el programa estadístico IBM $®$ SPSS $尺$ Statistics v25 (IBM, 2017), el cual permitió obtener puntuaciones, agrupaciones, medias y confiabilidad de los resultados conseguidos para su posterior análisis y discusión.

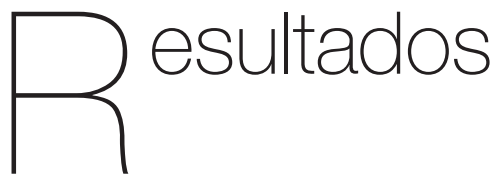

Los resultados contienen información referente a datos sociodemográficos, laborales y actitudes hacia el $\mathrm{CO}$, organizados en ocho dimensiones: relaciones personales, estilo de dirección, sentido de pertenencia, retribución, disponibilidad de recursos, estabilidad, claridad y coherencia de dirección, y valores colectivos, a través de la escala EDCO (Quevedo et al., 1999), siguiendo las instrucciones de uso de la misma, así como diligenciando el formato de consentimiento informado para los participantes.

Participaron voluntariamente en la investigación 85 funcionarios públicos de la Secretaría de Gobierno de Pasto, ubicados en la oficina principal, distribuidos en las dependencias: Oficina de Asesoría Jurídica (18.18\%), Subsecretaría de Justicia y Seguridad (15.45\%), Subsecretaría de Convivencia y Derechos Humanos (18.18\%) y Subsecretaría de Control (25.45\%).

Tabla 1.

Datos sociodemográficos y laborales de la muestra

\begin{tabular}{llc}
\hline \multirow{2}{*}{ Sexo } & Mujer & $31 \%$ \\
\hline Tiempo vincula- & Hombre & $69 \%$ \\
do a la entidad & 1 año o menos & $28.8 \%$ \\
\hline Tipo de vinculación & Planta temporal & $71.2 \%$ \\
\hline Total funcionarios & Contratista & $72.7 \%$ \\
\hline
\end{tabular}

Fuente: Elaboración propia

El perfil de la muestra evaluada fue: $69 \%$ hombres y $31 \%$ mujeres, con una vinculación laboral mayoritaria de tipo contratista $(72.7 \%)$ y planta temporal (27.3\%); además, el tiempo de adscripción en promedio fue de 3.9 años (ver Tabla 1).

A continuación, se presenta la información agrupada en cada una de las dimensiones que componen el CO general de la Secretaría de Gobierno de Pasto: 
Tabla 2

Clima general en la Secretaría de Gobierno

\begin{tabular}{|c|c|c|c|c|c|c|}
\hline \multirow{2}{*}{ Dimensión } & \multicolumn{6}{|c|}{ Frecuencia \% } \\
\hline & Muy alto & Alto & Medio & Bajo & Muy bajo & Total \\
\hline 1. Relaciones personales & 25.9 & 51.1 & 23.1 & 0 & 0 & 100 \\
\hline 2. Estilo de dirección & 49.2 & 44.2 & 6.6 & 0 & 0 & 100 \\
\hline 3. Sentido de Pertenencia & 10.4 & 24.7 & 16.2 & 37.2 & 11.5 & 100 \\
\hline 4. Retribución & 41.2 & 26.8 & 11.5 & 4.0 & 16.5 & 100 \\
\hline 5. Disponibilidad de recursos & 49.6 & 26.1 & 4.9 & 4.2 & 15.1 & 100 \\
\hline 6. Estabilidad & 21.4 & 12.7 & 21.2 & 25.6 & 19.1 & 100 \\
\hline 7. Claridad y coherencia de dirección & 46.1 & 24.9 & 12.0 & 5.6 & 11.3 & 100 \\
\hline 8. Valores colectivos & .2 & 18.1 & 50.4 & 31.3 & 0 & 100 \\
\hline
\end{tabular}

Fuente: Elaboración propia.

En la dimensión de relaciones personales se evidenció que el $51.1 \%$ de la población se mostró satisfecha, señalando que en el grupo de trabajo hay aceptación y unión entre sus integrantes; además, se tienen en cuenta sus opiniones y aportes (ver Tabla 2).

El estilo de dirección, entendido como la interacción de los jefes y colaboradores en torno a la participación, el estímulo, apoyo y confianza (Quevedo et al., 1999), fue percibido como muy satisfactorio y satisfactorio, con un 49.2\% y un 44.2\% respectivamente (ver Tabla 2).

El $48.7 \%$ de los funcionarios indicaron baja satisfacción en la dimensión sentido de pertenencia, resaltando que los beneficios y servicios de salud recibidos no satisfacen sus necesidades, seguido con el desacuerdo de la asignación salarial.

En cuanto a la retribución, entendida como el grado de equidad entre los beneficios derivados del trabajo (Quevedo et al., 1999), la mayoría de los funcionarios (68\%) se interesaron por el futuro de la entidad y la satisfacción de pertenecer a la misma.

Respecto a la dimensión disponibilidad de recursos, que comprende tanto el ambiente físico como la facilidad para el acceso a la información (Quevedo et al., 1999), el 75.7\% se sintió muy satisfecho en cuanto a la comodidad y la facilidad para desempeñar sus labores.

En la dimensión estabilidad la puntuación fue baja, con un 25.6\%, evidenciando principalmente que la entidad no brinda una estabilidad laboral por ser contratación temporal; además, no se tiene en cuenta el desempeño para la permanencia en el cargo.

En la dimensión claridad y coherencia de dirección, se encontró que la mayoría se muestra muy satisfecha con un porcentaje del $46.1 \%$, en la cual los directivos brindan información oportuna y clara respecto al cumplimento de las metas y los logros de la entidad.

Finalmente, en la dimensión valores colectivos, asumida como el grado de 
percepción en cuanto a cooperación, respeto y responsabilidad (Quevedo et al., 1999), se evidenció una puntuación media en la satisfacción de los colaboradores (50.4\%), resaltando el trabajo en equipo, así como en la relación entre los funcionarios de las otras dependencias.

Por otra parte, en la Tabla 3 se muestra la puntuación general del $\mathrm{CO}$ de la Secretaría de Gobierno de Pasto, de acuerdo a los parámetros de calificación establecidos por la escala EDCO (Quevedo et al., 1999).

Tabla 3

Calificación del CO

\begin{tabular}{lccc}
\hline Dimensión & $\mathrm{n}$ & Media & Total $\Sigma$ \\
\hline $\begin{array}{l}\text { 1. Relaciones personales } \\
\text { 2. Estilo de dirección }\end{array}$ & 85 & 20.1 & \\
$\begin{array}{l}\text { 3. Sentido de } \\
\text { Pertenencia }\end{array}$ & 85 & 14.3 & \\
$\begin{array}{l}\text { 4. Retribución } \\
\begin{array}{l}\text { 5. Disponibilidad } \\
\text { de recursos }\end{array}\end{array}$ & 85 & 18.6 & \\
$\begin{array}{l}\text { 6. Estabilidad } \\
\text { 7. Claridad y coherencia }\end{array}$ & 85 & 19.6 & 143 \\
de dirección & 85 & 14.6 & \\
8. Valores colectivos & 85 & 19.4 & \\
\hline
\end{tabular}

Nota: $\mathrm{n}=$ total funcionarios; Fuente: Elaboración propia.

El clima organizacional de la entidad obtuvo una puntuación de 143, interpretado como un nivel de satisfacción medio; asimismo, las dimensiones con puntuaciones más altas fueron: estilo de dirección y relaciones personales; con puntuaciones medias: claridad y coherencia de dirección, y disponibilidad de recursos. Por último, con puntuaciones bajas: sentido de pertenencia, valores colectivos y estabilidad.

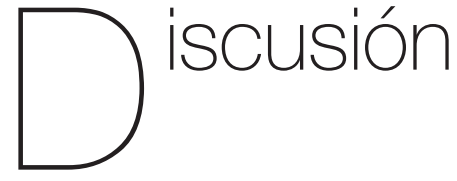

En primer lugar, los resultados obtenidos son similares a otros estudios realizados, coincidiendo especialmente en el grado de satisfacción del $\mathrm{CO}$ en las dimensiones: estilos de dirección y relaciones personales (Bacca \& Bravo, 2013; Rodríguez \& Torres, 2011; Rodríguez 2013; Fernández, Cobos \& Figueroa, 2015), las cuales puntuaron con nivel superior; por otra parte, sentido de pertenencia, valores colectivos y estabilidad (Fernández et al., 2015; Peña et al., 2015) registraron un nivel bajo.

De igual forma, la dimensión estilos de dirección que hace referencia al grado en que los jefes apoyan, estimulan y fomentan la participación de los colaboradores (Quevedo et al., 1999), obtuvo la mayor puntuación en el nivel de satisfacción del CO. Este resultado evidencia la relevancia de la dirección en las organizaciones, la cual orienta el desarrollo de los procesos que se llevan a cabo; no obstante, se debe comprender que todos los colaboradores desempeñan una función fundamental en la entidad y que sus roles pueden variar dependiendo de los procesos que se lleven a cabo, de este modo, se fomenta la participación y el trabajo colectivo para el cumplimiento de las metas (Blanco \& Caballero, 2005).

Lo anterior, además, resalta la estructura organizacional, ya que se permiten evidenciar dinámicas como: liderazgo, relaciones interpersonales, motivación, actitudes hacia el entorno laboral y comportamientos de los colaboradores, lo que, a 
la vez, influye sobre los valores, la cultura y el CO.

La segunda dimensión correspondiente a las relaciones personales, al ser percibida como favorable por la mayoría de los funcionarios, propicia un ambiente laboral adecuado, que fomenta el apoyo, la colaboración y el respeto; asimismo, disminuye problemas asociados con el ausentismo, la baja productividad, la motivación y la satisfacción en el desarrollo de las funciones (Bacca \& Bravo, 2013).

Las dimensiones sentido de pertenencia, valores colectivos y estabilidad, se relacionaron principalmente con los resultados de las investigaciones realizadas por Cardona (2016) y Escamilla et al. (2016), en las que se concluyó que las puntuaciones más bajas se obtuvieron en las variables relaciones en el trabajo, debido a la escasa comunicación entre las dependencias, en los beneficios obtenidos, en la asignación salarial y en el Plan de Seguridad Social (Salud, Pensión, ARL).

Con lo anterior, a pesar de que los servidores públicos tienen la responsabilidad de desarrollar y fortalecer las diversas políticas en beneficio de la ciudadanía, además de establecer una relación permanente entre la entidad pública y los ciudadanos (Pliscoff, 2006), se han evidenciado falencias en las acciones dirigidas hacia el mejoramiento de la calidad de vida laboral de los funcionarios y en los beneficios recibidos (Sanabria, 2016), resaltando del estudio que, según los servidores públicos, las acciones en cuanto a los servicios de salud, la asignación salarial y las políticas en la contratación fueron escasas e insatisfactorias, puesto que, al ser el contrato por prestación de servicios, las recompensas obtenidas son distintas y en menor proporción, comparándolas con funcionarios de planta, lo que genera baja percepción de estabilidad.

Teniendo en cuenta que los beneficios personales obtenidos por los colaboradores son elementos importantes que inciden sobre el CO (Díaz \& Carrasco, 2018), respecto al Plan de Seguridad Social, en el territorio Colombiano, se encontró que seguramente existe una relación con el tipo de contratación laboral, debido a que la mayoría del personal son contratistas vinculados a la entidad en condición de prestación de servicios temporales, siendo responsables de cubrir los costos en su sistema de salud mediante el aporte al Sistema de Seguridad Social Integral, según lo establecido por el Estado en el Plan Nacional de Desarrollo (PND) a través el Decreto 1273 de 2018; razón por la cual es un indicador de insatisfacción respecto a la recompensa recibida.

Otro aspecto de interés son los valores colectivos, en los cuales se evidenció baja puntuación, especialmente en lo referente al medio interno y las relaciones con las demás dependencias. En este caso, de acuerdo con Boria et al. (2013), los valores son fundamentales en el desarrollo de las actitudes y conductas de los colaboradores hacia su entorno laboral, puesto que los trabajadores, al asumir el sistema de valores de su entidad, promueven la identidad y cultura de ésta, reflejándose en las relaciones personales y el manejo de los conflictos; por lo tanto, los valores de una organización deben ser claros, aceptados y compartidos, reflejando los intereses de todos los integrantes de la organización y no pueden ser vistos como un elemento más de la filosofía de la entidad (Marchant \& Del Rio, 2008). 
En conclusión, en el estudio realizado, los funcionarios indicaron sentirse medianamente satisfechos con el CO de la Secretaría de Gobierno de Pasto, puesto que se evidenciaron aspectos positivos relacionados con la dirección de la entidad y los recursos que se brindan para el desempeño de sus funciones, sin embargo, es necesario fortalecer los aspectos como la estabilidad laboral, los beneficios obtenidos y las relaciones entre las distintas dependencias, identificando aquellas que requieran mayor atención, analizando las posibles estrategias que permitan su fortalecimiento e interviniendo oportunamente para lograr un mejoramiento y aprendizaje continuo que fortalezca el CO de la entidad y así se potencialice el capital humano.

Se señala que no existen estudios realizados en esta secretaría debido a las políticas, las condiciones de privacidad y el manejo de la información en la entidad pública, por lo tanto, esta investigación permite identificar elementos relevantes para futuros estudios e intervenciones sobre esta temática y su población, resaltando especialmente que en las organizaciones se pueden evidenciar micro climas distintos, a pesar de estar bajo las mismas políticas o estructura, especialmente si están disgregadas (Álvarez, 1992), de manera que se hace necesario que las organizaciones fortalezcan su entorno laboral teniendo en cuenta los cambios en su contexto a partir de la globalización económica, e implementen el uso de la nueva tecnología en la creación de instrumentos innovadores, adaptados a las necesidades propias de la entidad y su entorno cultural (Dávila, Escobar \& Mullet, 2011).
Finalmente, en la investigación se presentaron algunas limitaciones correspondientes al acceso a la población para dicho estudio, la variación en el número de funcionarios por cada una de las subsecretarías, así como la descentralización en las dependencias adscritas a la Secretaría de Gobierno; es por ello que la investigación se realizó únicamente en la sede principal que reunía al mayor número de funcionarios. Se sugiere, para posteriores estudios, la intervención sobre el CO desde los distintos enfoques de la psicología, especialmente desde la psicología cognitiva y conductual, analizando los distintos procesos cognitivos y su funcionamiento, para comprender el comportamiento humano que, a su vez, puede incidir en las estructuras y procesos organizacionales.

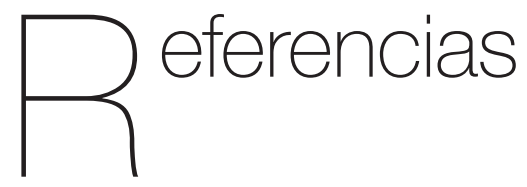

Aguilar, S. (2005). Fórmulas para el cálculo de la muestra en investigaciones de salud. Salud en Tabasco, 11(1-2), 333-338. Recuperado de https://www.redalyc.org/ articulo.oa?id=487/48711206

Álvarez, G. (1992). El Constructo Clima Organizacional: Concepto, teorías, investigaciones y resultados relevantes. Revista Interamericana de Psicología Ocupacional, 11(1-2), 27-30. Recuperado de http://www.redalyc.org/ pdf/761/76111485014.pdf

Bacca, A., \& Bravo, S. (2013). Incidencia de una estrategia de fortalecimiento en trabajo en equipo en el clima organizacional de 
una entidad del sector público. (Tesis de Pregrado). Universidad de Nariño, San Juan de Pasto, Nariño, Colombia.

Blanco, A., \& Caballero, A. (2005). Psicología de los grupos. Madrid, España: PearsonPrentice hall.

Betancourt, A., \& Caballero, A. (2010). Lafunción de los Recursos Humanos y su aporte a la empresa actual. Revista digital sociedad de la información, 21, 1-5. Recuperado de http://www.sociedadelainformacion. com/21/recursos.pdf

Boria, S., Crespi, M., García, A., \& Vizuete, E. (2013). Los valores compartidos en la empresa española. Universia Business Review, (37), 68-85. Recuperado de https://www.redalyc.org/articulo. oa?id=433/43325648004

Bustamante, M., Grandón, M., \& Lapo, M. (2015). Caracterización del clima organizacional en hospitales de alta complejidad en Chile, 31(137), 432-440. Recuperado de https://www.redalyc.org/ articulo.oa?id=212/21243557009

Bravo, E. (2009). Tendencias en el mercado de trabajo a partir de la globalización. Otra modernidad y otro fracaso latinoamericano. Fermentum. Revista Venezolana de Sociología y Antropología, 19(55), 215-234. Recuperado de https://www.redalyc.org/ articulo.oa?id=705/70517703003

Cardona, A. (2016). Medición del clima Organizacional en la Alcaldía de Palestina. (Tesis de pregrado). Universidad Tecnológica de Pereira, Colombia.

Charry, H. (2018). Lagestión de la comunicación interna y el clima organizacional en el sector público. Comuni@cción, 9(1),
25-34. Recuperado de http://www. scielo.org.pe/scielo.php?script=sci_ arttext\&pid=\$2219-2018000100003

Chaves, L., Grijalba, M., Bárcenas, D., Matabanchoy, S., \& Zambrano, C. (2017). Actitudes hacia la calidad de vida laboral en trabajadores control de vía de transporte urbano. Tendencias, 18(2), 69-85. https:// doi.org/10.22267/rtend.171802.77

Chiavenato, I. (2001). Administración de recursos humanos. México: McGraw-Hill.

Dávila, D., Escobar, A., \& Mullet, L. (2011). El clima organizacional en las empresas innovadoras del siglo XXI. Informes Psicológicos, 12(2), 105-115. Recuperado de https://revistas.upb.edu.co/index. php/informespsicologicos/article/ view/1723/1837

Decreto 1273 (2018). Ministerio de Salud y Protección Social. [En línea] Recuperado de http://es.presidencia.gov.co/normativa/ normativa/DECRETO\%201273\%20 DEL\%2023\%20DE\%20JULIO\%20DE\%20 2018.pdf

Díaz, F., \& Carrasco, M. (2018). Efectos del clima organizacional y los riesgos psicosociales sobre la felicidad en el trabajo. Contaduría y Administración, 63(4), 1-14. https://dx.doi. org/10.22201/fca.24488410e.2018.1142

Escamilla, P., Núñez, J., \& Gómez, A. (2016). Clima Organizacional y sector de pertenencia: un análisis de la percepción de los empleados de entidades deportivas. Revista de Psicología del Deporte, 25(1), 7376. Recuperado de https://www.redalyc. org/articulo.oa?id=2351/235146293017</ a> 
Fernández, R., Cobos, P., \& Figueroa, M. (2015). Evaluación del clima organizacional en un centro de rehabilitación y educación especial. Revista Cubana de Salud Pública, 41(4), 593-602. Recuperado de https://www.redalyc.org/articulo. oa?id=214/214435

Garzón, M. (2005). El desarrollo organizacional y el cambio planeado. Bogotá: Centro Editorial Universidad del Rosario.

Gómez, C. (2004). Diseño, construcción y validación de un instrumento que evalúa clima organizacional en empresas colombianas, desde la teoría de respuesta al ITEM. Acta Colombiana de Psicología, (11), 97-113 Recuperado de https://www. redalyc.org/articulo.oa?id=798/79801108

Goncalves, A. (2000). Fundamentos del clima organizacional. Recuperado de http:// www.calidad.org/articles/ dec97/artdec97. htm

IBM. (2017). IBM SPSS Statistics for Windows, Version 25.0. Armonk, NY: IBM Corp.

Ley 1090 (2006). "De la profesión de Psicología". [En línea] Recuperado de http://www.psicologiaprospectiva.com/ introley1090.html

Marchant, L., \& Del Rio, A. (2008). Gestión estratégica de la diversidad cultural en las organizaciones. Universidad de Viña del Mar. Chile.

Montoya, C., \& Boyero, M. (2016). El recurso humano como elemento fundamental para la gestión de calidad y la competitividad organizacional. Revista Científica "Visión de Futuro", 20(2), 1-20. Recuperado de https://www.redalyc.org/articulo. oa?id=3579/357947335001
Peña, R., Hernández, D., Vélez, A., G, M., Reyes, M., \& Ureña, E. (2015). Clima organizacional de equipos directivos y su relación con los resultados en la atención a la salud. Salud Pública de México, 57(6), 528-536. Recuperado de https://www.redalyc.org/ articulo.oa?id=106/10642748007

Pérez, D., \& Castillo, J. (2016). Capital humano, teorías y métodos: importancia de la variable salud. Economía, Sociedad y Territorio, 16(52), 651-673. Recuperado de http://www.redalyc.org/articulo. oa?id=11146910004

Plan Nacional de Desarrollo (2018). Plan Nacional de Desarrollo 2018-2022. [En línea] Recuperado de https://colaboracion. dnp.gov.co/CDT/Prensa/ResumenPND2018-2022-final.pdf

Pliscoff, C. (2006). Reflexiones sobre el "Proyecto de investigación para formular bases de diseño, implementación y evaluación de una política integral que promueva la innovación y la efectividad en la administración y gestión del talento humano en el sector público", en la administración pública colombiana. Colombia: Ediciones Uniandes.

Quevedo, A., Echeverri, S., \& Sanabria, B. (1999). Escala de Clima Organizacional (EDCO). Fundación Universitaria Konrad Lorenz, Bogotá, Colombia.

Rodríguez, N., \& Torres, A. (2011). El clima organizacional como facilitador para la innovación en el proyecto comedores comunitarios institucionales. (Tesis de Pregrado). Universidad Jorge Tadeo Lozano, Bogotá, Colombia.

Rodríguez, R. (2013). Percepción individual de clima organizacional y desempeño laboral 
en los servidores públicos de carrera administrativa del ICBF sede regional Nariño. (Tesis de pregrado). Universidad de Nariño, San Juan de Pasto, Colombia.

Salas, C., \& Ortiz, A. (2008). Presentación del modelo "Alpha" para el diagnóstico y evaluación del clima organizacional aplicado a la secretaría de educación y cultura de Nariño. (Tesis de Pregrado). Universidad de Nariño, San Juan de Pasto, Colombia.

Salas F., Ortiz, P., \& Márquez, A. (2016). Lineamientos de política de empleo público y de gestión de recursos humanos 20152025. Colombia: Kimpres S.A.S.
Sanabria, P. (2016). ¿Cómo poner en marcha un modelo de gestión estratégica del talento humano para el sector público colombiano? Colombia: Ediciones Uniandes.

Torres, M. (2015). Análisis institucional de la evolución de la política pública de gestión del talento humano en Colombia. Bogotá: Universidad de los Andes.

Vizcarra, M., Llaja V., Limo, C., \& Talavera, J. (2015). Clima laboral, Burnout y Perfil de Personalidad: Un estudio en personal asistencial de un Hospital Público de Lima. Informes Psicológicos, 15(2), 111-126. http:// dx.doi. org/10.18566/infpsicv15n2a06 